\title{
Effets du Précédent Cultural de L'arachide (Arachis Hypogaea L.) et de la Fumure Minérale sur la Production du Coton (Gossypium Hirsutum L.)
}

\author{
N'Goran Kouadio Emmanuel \\ Centre National de Recherche Agronomique (CNRA), Abidjan, \\ Côte d'Ivoire \\ Ama Tamia Joséphine Epse Abina \\ Université Nangui Abrogoua (UNA) Abidjan, Côte d'Ivoire, \\ UFR Sciences et Gestion de l'Environnement, Abidjan \\ Yoboue Ahou Natachat Epse Kouakou \\ Université Nangui Abrogoua (UNA) Abidjan,Côte d'Ivoire, \\ UFR Sciences et Gestion de l’Environnement, Abidjan
}

Doi:10.19044/esj.2021.v17n34p260

Submitted: 19 April 2021

Accepted: 02 September 2021

Published: 30 September 2021
Copyright 2021 Author(s)

Under Creative Commons BY-NC-ND 4.0 OPEN ACCESS

Cite As:

N’Goran K.E., Abina A.T.J.E. \& Kouakou Y.A.N.E. (2021). Effets du Précédent Cultural de L'arachide (Arachis Hypogaea L.) et de la Fumure Minérale sur la Production du Coton (Gossypium Hirsutum L.). European Scientific Journal, ESJ, 17(34), 260.

https://doi.org/10.19044/esj.2021.v17n34p260

\section{Résumé}

Pour restaurer et améliorer la fertilité des sols en culture de coton, l'arachide (Arachis hypogaea L) a été utilisée comme précédent cultural. L'expérimentation a été faite suivant un dispositif en blocs de Fisher avec quatre (4) répétitions et cinq (5) traitements, sur deux années (2018 et 2019). Les résultats ont montré que l'arrière effet de l'arachide (la biomasse et l'azote résiduel issue de la symbiose entre la légumineuse et les bactéries) a amélioré la croissance et le développement végétatif des plants de cotonnier. L’impact de cet apport organique s'est traduit par des plants de cotonniers statistiquement plus grand, (hauteur supérieur à $100 \mathrm{~cm}$ ) pour le traitement T5 (précédent arachide + dose complet d'engrais). Le traitement T4 (précédent arachide $+1 / 2$ dose d'engrais) quant à lui a généré un nombre moyen de capsules supérieur à 8 à la seconde campagne. Il ressort également que le précédent arachide associé à la dose complète et à la demi dose de fumure minérale améliore mieux le rendement du coton-graine. Des gains de 3 à 14 
\% à la 1ère campagne et 19 à 58 \% à la deuxième campagne ont été enregistrés . Ainsi, la rotation arachide/coton est une bonne pratique, à encourager.

Mots clés : Coton, Arachide, Biomasse, Fumure Minérale, Rendement

\title{
Effects of Peanut (Arachis Hypogaea L.) Cultural Background and Mineral Manure on Cotton (Gossypium Hirsutum L.) Production
}

\author{
$N$ 'Goran Kouadio Emmanuel \\ Centre National de Recherche Agronomique (CNRA), Abidjan, \\ Côte d'Ivoire \\ Ama Tamia Joséphine Epse Abina \\ Université Nangui Abrogoua (UNA) Abidjan, Côte d’Ivoire, \\ UFR Sciences et Gestion de l'Environnement, Abidjan \\ Yoboue Ahou Natachat Epse Kouakou \\ Université Nangui Abrogoua (UNA) Abidjan,Côte d’Ivoire, \\ UFR Sciences et Gestion de l'Environnement, Abidjan
}

\begin{abstract}
To restore and improve soil fertility in cotton cultivation, peanut (Arachis hypogaea L) was used as a previous crop. The experimentation was done in a Fisher block design with four (4) replications and five (5) treatments, over two years (2018 and 2019). The results showed that the after-effect of the peanut (biomass and residual nitrogen from the symbiosis between the legume and the bacteria) improved the growth and vegetative development of the cotton plants. The impact of this organic fertilizer resulted in statistically larger cotton plants (height greater than $100 \mathrm{~cm}$ ) for treatment T5 (previous groundnut + full dose of fertilizer). Treatment T4 (previous groundnut $+1 / 2$ dose of fertilizer) generated an average number of bolls greater than 8 in the second season. It also appears that the groundnut precedent associated with different doses of mineral fertilizer improves seed cotton yields. Gains of 3 to $14 \%$ in the first season and 19 to $58 \%$ in the second season were recorded. Thus, the groundnut/cotton rotation is a good practice that should be encouraged.
\end{abstract}

Keywords: Cotton, Groundnut, Biomass, Mineral Manure, Yield

\section{Introduction}

Le cotonnier, plante cultivée en Afrique Subsaharienne, produit la fibre textile naturelle la plus exploitée dans le monde. Cette fibre constitue la 
matière première principale en industrie textile (Berti et al., 2006). Depuis les années 50 , la culture du coton contribue à l'épanouissement socio-économique de la population, des zones de savane de Côte d'Ivoire (Ochou et al., 2003). Cependant, la culture du coton, est soumise à des contraintes telles que l'irrégularité et la mauvaise répartition des pluies, le non-respect des itinéraires techniques, les attaques des ravageurs et autres maladies du cotonnier. A ceci s'ajoute la dégradation physique et chimiques des sols (Bado, 2002 ; Adingra, 2011 ; Samba et al, 2013). Cet appauvrissement des sols est le résultat d'une démographie croissante limitant l'étendue et la durée des jachères jadis pratiquées pour améliorer les sols (Akédrin et al., 2010; Coulibaly et al., 2012). Pour résoudre le problème d'appauvrissement des sols, les producteurs utilisent les intrants chimiques pour augmenter significativement la productivité des cultures. Cependant, l'usage exclusif des engrais chimiques à long terme ne peut assurer la sécurité des sols et une production de qualité acceptable. Des études ont montré que les engrais chimiques acidifient les sols; ce qui a un impact négative sur le développement du système racinaire de la plupart des plantes (FAO, 2015).

Il apparait, de ce fait, nécessaire de concevoir des systèmes culturaux plus productifs et plus durables qui tiennent compte de la restauration des sols et de la qualité de la production (Coulibaly, 2012). En effet, de nombreuses cultures associées aux légumineuses alimentaires ont donné de résultats satisfaisants (N’Goran et al., 2011 ; Konaté et al., 2012). En générale, les légumineuses restaurent les sols par l'azote résiduel qu'elles laissent dans le sol en fin de cycle. Et cet azote est exploitable par d'autres cultures (Akédrin et al., 2010 ; Gbakatchetche et al. 2010). Elles offrent donc de bonnes perspectives de valorisation des cultures en vue d'une intensification écologique (Hinsinger, 2012). Le maintien de la biomasse des légumineuses au sol, peut favoriser l'agrégation et la stabilité structurale des sols agricoles. La biomasse des légumineuses se décompose facilement et restitue des éléments nutritifs au sol pour les autres cultures (Anonyme, 2013). Leur utilisation permet d'augmenter le revenu brut du paysan en réduisant les charges en engrais (Carsky et al., 2003). Elles apparaissent donc nécessaires pour une agriculture durable (Vertès et al., 2015). L'usage satisfaisant des légumineuses en association ou en avant-culture avec d'autres plantes a été longtemps prouvé. Cependant, l'étude de l'effet des légumineuses alimentaires sous cotonnier reste peu explorée.

Cette étude permettra d'évaluer l'effet conjugué de la biomasse produite par l'arachide et de la fumure minérale sur la production du coton. 


\section{Matériel et Méthodes}

Site d'étude

L'étude a été faite au Centre National de Recherche Agronomique (CNRA) à Bouaké, ville située au Centre de la Cote d'Ivoire, dont les coordonnées géographiques sont $7^{\circ} 69^{\prime} \mathrm{N}$ et $5^{\circ} 03^{\prime} \mathrm{W}$ et $376 \mathrm{~m}$ d'altitude.

La ville de Bouàké a un climat de type Soudanien avec deux saisons sèches et deux saisons de pluies peu marquées (Ouattara, 2001). Dans cette zone, l'ensoleillement est plus constant et la précipitation moyenne est d'environ $1100 \mathrm{~mm}$ par an repartie de façon irrégulière sur toute l'année. Sur les deux années consécutives (2018 et 2019), la saison de pluie a débuté en février pour prendre fin en novembre. Les pics de pluviométrie ont été centrés respectivement sur le mois de septembre (331 mm) en 2018 et le mois d'Août (264 mm) en 2019. La saison sèche s'est étalée de novembre à janvier (Figure 2). La température moyenne a été de $26,2^{\circ} \mathrm{C}$. La ville est située dans la zone de transition forêt-savane (N'Goran, 2008). Le relief est très peu accidenté avec une altitude moyenne de $200 \mathrm{~m}$. Les sols de la région sont généralement peu profonds et légers. Ce sont des ferasols remaniés, à texture sablo-argileuse ou argilo-sableuse, avec un bon drainage. Ces sols acides $(\mathrm{pH}=5-6)$ sont pauvres en matière organique (Yoro, 2000 ; N'Goran et al., 2018). Mais, ils présentent souvent une pellicule de battance qui favorise le ruissellement.

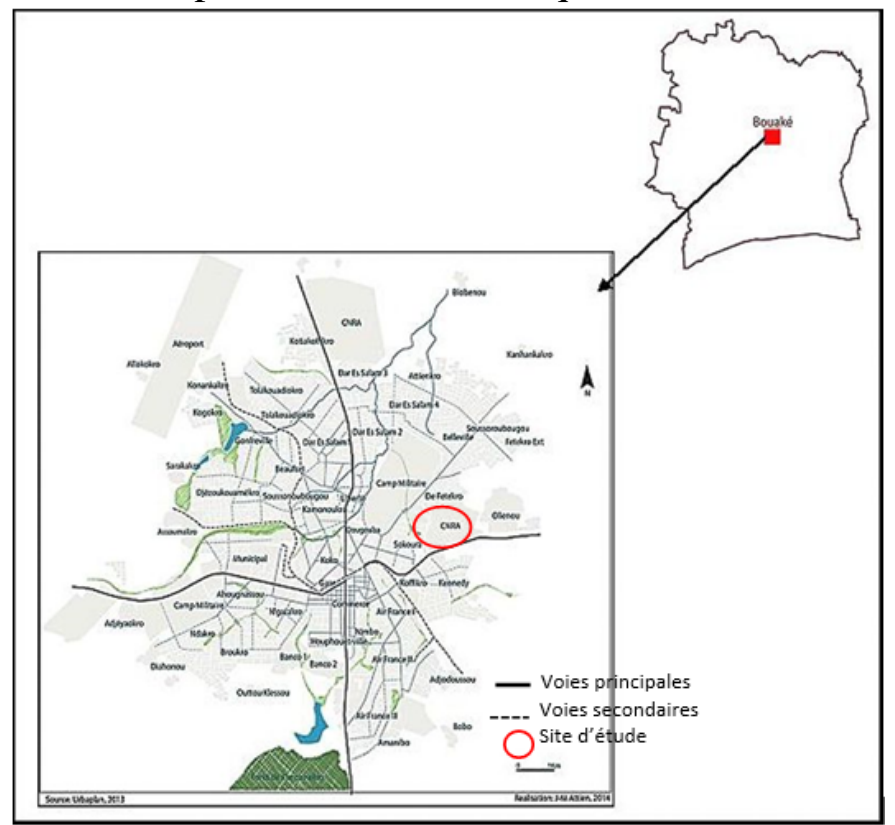

Figure 1. Site d'étude localisé dans la ville de Bouaké 


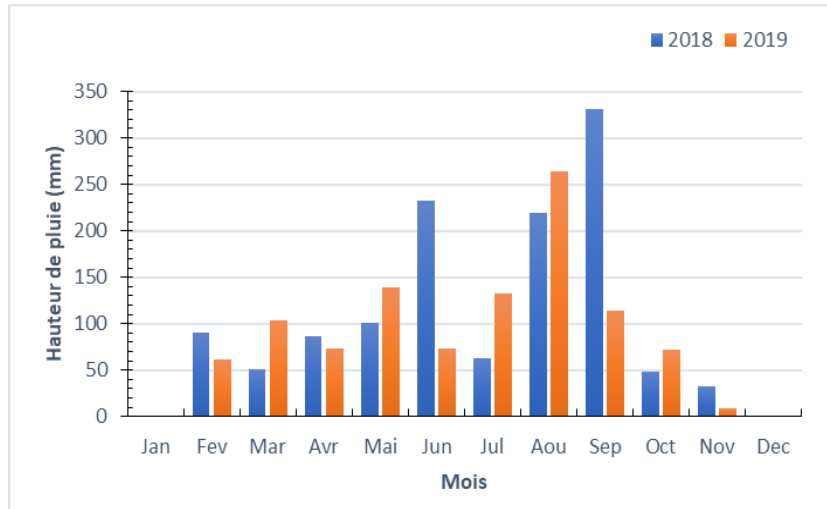

Figure 2. Histogrammes comparatives des pluviométries (2018 et 2019)

\section{Matériel végétal}

Le matériel végétal est constitué de semences de coton (Gossypium hirsutum L.) et de celles de l'arachide (Arachis hypogaea). Le coton utilisé est la variété sicama $\mathrm{V}-1$, issue de la collection des ressources génétiques du CNRA sélectionnée en 2006. Cette variété a une hauteur moyenne de $135 \mathrm{~cm}$ et s'adapte aux conditions climatiques et édaphiques de la Côte d'Ivoire. Elle se caractérise par un rendement pouvant atteindre 4 t/ha en coton-graine. Elle a une bonne qualité de la fibre, et un taux de fibre à l'égrenage très élevé (45 \%). L'arachide, de la variété 8-20 est issue de la sélection 2008-2009 du CNRA. Cette variété ayant un cycle de 90 jours est très productive avec un rendement en gousses pouvant atteindre $4 \mathrm{t} / \mathrm{ha}$. Elle est tolérante à la rosette et à la cercosporiose qui sont deux maladies redoutables en culture d'arachide.

\section{Matériel technique et chimique}

Le matériel technique pour la culture du coton est composé, entre autres, de cordes pour tracer les parcelles, de dabas pour le labour, le semis et le sarclage, d'étiquettes pour identifier les parcelles élémentaires (traitements), d'équipement de protection individuel (EPI) pour le traitement insecticides.

Le matériel chimique utilisé est un fertilisant minérale composé de deux types d'engrais que sont le NPKSB, utilisé comme engrais de fond et l'urée (46 \% de N) comme engrais de couverture. Les produits phytosanitaires pour la protection des cotonniers sont Caïman B 19, IBIS A 52, polytrine C365 et Thian 250.

\section{Dispositif expérimental}

L'essai a été conduit suivant un dispositif en blocs de Fisher comportant quatre répétitions et cinq traitements qui sont :

- $\quad$ T1 : Précédent jachère naturelle (JN) de deux ans (Témoin absolu); 
- T2 : Précédent jachère naturelle de deux ans + 200 kg/ha NPK + $50 \mathrm{~kg} /$ ha d'Urée (JN + DV);

- T3 : Précédent légumineuse +0 kg d'engrais coton ( $\mathrm{L}+0 \mathrm{DV})$;

- T4 : Précédent légumineuse $+1 / 2$ dose $(100 \mathrm{~kg} / \mathrm{ha}) \mathrm{NPK}+1 / 2$ dose (25 kg/ha) d'Urée (L+1/2 DV);

- T5 : Précédent légumineuse $+200 \mathrm{~kg} / \mathrm{ha}$ NPK $+50 \mathrm{~kg} / \mathrm{ha}$ d’Urée ( $\mathrm{L}+\mathrm{DV})$.

Les traitements ont été répartis de façon aléatoire dans chaque bloc. Les parcelles élémentaires (10 $\mathrm{m}$ de long et 6,4 $\mathrm{m}$ de large) sont constituées de 8 lignes de coton de $10 \mathrm{~m}$ de long. Une distance de $2 \mathrm{~m}$ sépare deux blocs.

\section{Conduite des cultures}

Le labour et le pulvérisage du sol ont eu lieu en début du mois de Mars. Cela a été suivi d'un léger hersage pour aplanir le terrain, afin de déterminer les parcelles élémentaires. L'arachide a été mise en place en avant culture en mars. Le semis a été fait selon des écartements de $50 \mathrm{~cm}$ entre les lignes et 20 $\mathrm{cm}$ entre les poquets. Un démariage à deux plants a été fait après le semis, soit une densité maximale de 200000 plants à l'hectare. Deux sarclages ont été effectués avant la récolte. La récolte a été faite en fin juin.

Le semis de coton a été fait dans la $1^{\text {ere }}$ décade de juillet (entre $1^{\text {er }}$ et 10 juillet). Il s'est fait selon un écartement de $80 \mathrm{~cm}$ entre les lignes et $30 \mathrm{~cm}$ entre les poquets, soit une densité maximale de 83500 plants à l'hectare, avec deux plants par poquet. Les consignes générales de culture cotonnière ont été respectées : application d'herbicide après semis, démariage 15 jours après la levée (JAL), resemis, sarclages, apport d'engrais NPK (15 à $20 \mathrm{JAL}$, au moment du 1er sarclage) selon les différents traitements et buttage plus épandage d’Urée (45 JAL), à l'apparition des premières fleurs.

\section{Collecte et analyse des données}

\section{Détermination de la biomasse sèche}

La détermination de la biomasse sèche de l'arachide a été estimée à 90 jours après semis (JAS). Les plants d'arachide des carrés de rendement $\left(4 \mathrm{~m}^{2}\right)$ de chaque parcelle élémentaire ont été déterrés. Après la récolte des gousses, la biomasse a été séchée à l'air libre, puis pesée pour en déterminer le poids total et le rendement par hectare.

$$
R D T=\frac{M b \times 10000}{S}
$$

Avec RDT en $\mathrm{kg} / \mathrm{ha}, \mathrm{M}_{\mathrm{b}}=$ masse de la biomasse en $\mathrm{kg}$ et $\mathrm{S}=$ surface récoltée en $\mathrm{m}^{2}$.

Un échantillon composite a été prélevé, puis broyé pour l'analyse des éléments minéraux tels que l'azote $(\mathrm{N})$, le phosphore $(\mathrm{P})$, le potassium $(\mathrm{K})$, le calcium (Ca) et le magnésium (Mg) au laboratoire. 


\section{$>$ Evaluation des paramètres agro-morphologiques du cotonnier}

Les observations portant sur les paramètres morphologiques ont été réalisées sur 10 plants (bien développés) choisis au hasard sur les 4 lignes centrales des parcelles élémentaires suivant la méthode de la diagonale. Les paramètres morphologiques qui ont été évalué sont :

\section{- Hauteur des plants de cotonniers}

La hauteur des cotonniers s'est faite par mesure de la tige principale, à parti des nœuds cotylédonaires, à 30, 60, 90 JAL et à la récolte (120 JAL).

- Nombre de capsules vertes

Le nombre de capsules vertes a été déterminé par le comptage des capsules vertes sur dix (10) plants choisis, aux $70^{\mathrm{e}}, 90^{\mathrm{e}}$ et $110^{\mathrm{e}}$ jours après levée (JAL).

\section{- Taux de mortalité}

Le taux de mortalité des cotonniers a été évalué par comptage du nombre de plants après le démariage (NPD) et à la récolte (NPR), sur quatre lignes centrales de $8 \mathrm{~m}$ de long. Il est déterminé à partir de la formule suivante:

$$
\mathrm{TxM}=\frac{(\mathrm{NPD}-\mathrm{NPR})}{\mathrm{NPD}} \times 100
$$

\section{- Rendement en coton-graine}

La récolte de coton-graine s'est faite sur une séquence de 4 lignes centrales de $8 \mathrm{~m}$ par parcelle élémentaire, soit une surface de 25,6 $\mathrm{m}^{2}$ (4 lignes x $0.8 \mathrm{~m}$ x $8 \mathrm{~m}$ ). Elle s'est faite à $50 \%$ puis à $100 \%$ d'ouverture et a été pesée. Le rendement en coton-graine, exprimé en $\mathrm{kg} / \mathrm{ha}$, a été déterminé selon la formule:

$$
\text { Rendement }(\mathrm{Kg} / \mathrm{ha})=\frac{(\text { Poids récolté }(\mathrm{kg}) \times 10000)}{\text { Superficie récoltée }\left(\mathrm{m}^{2}\right)}
$$

- Efficience agronomique (AE) des fertilisants

Elle est le rapport entre le rendement supplémentaire par rapport au traitement témoin obtenu grâce à la quantité d'engrais apportée (Akassimadou et al, 2017). Elle est obtenue par la formule :

$$
\begin{aligned}
& \text { Efficience } \\
& \text { Rendement avec engrais }- \text { rendement sans engrais }
\end{aligned}
$$

\section{Méthodes statistiques}

Les logiciels EXCEL et STATISTICA 7.1 ont été utilisés pour le traitement des données. EXCEL a permis d'enregistrer les données brutes dans des matrices. Avec STATISTICA 7.1, l'analyse de variance (ANOVA) à deux (2) critères a été observée. La comparaison des moyennes a été effectuée au seuil de 5\%. En cas de différence significative, le test de Student-NewmanKeuls a servi à déterminer les groupes homogènes. 


\section{Résultats}

\section{Mobilisation d'éléments minéraux produite par l'arachide sur les deux années}

La quantité de matière sèche produite la $1^{\text {ère }}$ année (8469 kg/ha) a été moins que celle de la $2^{\mathrm{e}}$ année (10083 kg/ha). La teneur des minéraux à la $2^{\mathrm{e}}$ année a été également supérieure à celle obtenue à la $1^{\text {ère }}$ année quel que soit l’élément minéral étudié (Tableau I).

Tableau I. Concentration en éléments minéraux et quantité d'éléments minéraux (kg/ha) mobilisée par biomasses sèches d'arachide

\begin{tabular}{|c|c|c|c|}
\hline Minéraux & $\begin{array}{c}\text { Concentration } \\
(\% \mathrm{~ms})\end{array}$ & $\begin{array}{c}1^{\text {ère }} \text { année } \\
(8469 \mathrm{Kg} / \mathrm{ha})\end{array}$ & $\begin{array}{c}2^{\text {ère }} \text { année } \\
(10083 \mathrm{Kg} / \mathrm{ha})\end{array}$ \\
\cline { 3 - 4 } & 2,52 & 213,42 & 254,09 \\
\hline $\mathrm{N}$ & 0,285 & 24,13 & 28,74 \\
\hline $\mathrm{P}$ & 2,427 & 205,54 & 244.71 \\
\hline $\mathrm{K}$ & 1,723 & 145.92 & 173,73 \\
\hline $\mathrm{Ca}$ & 0,484 & 40,99 & 48,8 \\
\hline $\mathrm{Mg}$ & \multicolumn{2}{|c}{} \\
\hline
\end{tabular}

\section{Effet du précédent cultural de l'arachide et de la fumure minérale sur les paramètres agro-morphologiques du cotonnier}

\section{Densité des plants au démariage, à la récolte et taux de mortalité}

Le tableau II illustre le nombre de plants de cotonnier au démariage et à la récolte, ainsi que le taux de mortalité en fonction des traitements, sur les deux années.

Le nombre de plants au démariage et à la récolte a varié respectivement de 72363 à 77 148, et de 60059 à 68 164, la 1ère année. Celui de la 2e année a varié respectivement de 63906 à 80 313, et de 59219 à 76 797. Les résultats obtenus ont été statistiquement identique pour les deux années de campagne. Toutefois, pour les cotonniers issus des précédents arachides (T3, T4 et T5) les valeurs numériques des densités de cotonniers ont été supérieures aux traitements T1 et T2 à l'hectare. Quant au taux de mortalité des plantes de cotonnier, il a varié de 10,24 à 17,06 \%, la $1^{\text {ère }}$ année et de 1,67 à 8,23\%, la 2e année. Les valeurs obtenues dans les différents traitements ont été statistiquement identique, mais les cotonniers issus des précédents jachères (T1 et T2) ont eu les taux de mortalité supérieurs aux précédents arachide (T3, T4 et T5)

Tableau II. Nombre de plants de cotonniers au démariage, à la récolte et taux de mortalité

\begin{tabular}{|c|c|c|c|c|}
\hline \multirow[t]{2}{*}{ Années } & \multirow[b]{2}{*}{ Traitements } & \multicolumn{2}{|c|}{ Nombre de plants de cotonnier par ha } & \multirow{2}{*}{$\begin{array}{l}\text { Taux de } \\
\text { mortalité } \\
\text { (p.c.) }\end{array}$} \\
\hline & & $\begin{array}{l}\text { Après } \\
\text { démariage }\end{array}$ & A la récolte & \\
\hline & $\mathrm{T} 1(\mathrm{JN})$ & $72363 \pm 2229 a$ & $60059 \pm 3044 a$ & $17,06 \pm 2,82 a$ \\
\hline & $\mathrm{T} 2(\mathrm{JN}+\mathrm{DV})$ & $73730 \pm 1778 a$ & 62 695 $\pm 2179 a$ & $14,98 \pm 1,79 a$ \\
\hline & T3 (L+ 0 DV) & $76074 \pm 2159 a$ & $68164 \pm 789 a$ & $10,24 \pm 2,03 a$ \\
\hline
\end{tabular}




\begin{tabular}{|c|c|c|c|c|}
\hline \multirow{5}{*}{$\begin{array}{l}2018- \\
2019\end{array}$} & T4 (L+ 1/2 DV) & $75879 \pm 665 a$ & $65820 \pm 977 a$ & $13,27 \pm 0,71 a$ \\
\hline & T5 (L+ DV) & $77148 \pm 2020 a$ & $66406 \pm 2635 a$ & $13,97 \pm 2,20 a$ \\
\hline & Moyenne & $75039 \pm 840$ & $64629 \pm 1073$ & $13,90 \pm 0,96$ \\
\hline & Probabilité (P) & 0,288 & 0,651 & 0,248 \\
\hline & Significativité & NS & NS & NS \\
\hline \multirow{8}{*}{$\begin{array}{l}2019- \\
2020\end{array}$} & $\mathrm{~T} 1(\mathrm{JN})$ & $64922 \pm 5673 a$ & $63203 \pm 5991 a$ & $2,68 \pm 0,68 a$ \\
\hline & $\mathrm{T} 2(\mathrm{JN}+\mathrm{DV})$ & $63906 \pm 7016 a$ & $59219 \pm 8138 a$ & $8,23 \pm 3,31 a$ \\
\hline & T3 (L+ 0 DV) & $80313 \pm 2532 a$ & $76797 \pm 2269 a$ & $4,30 \pm 1,90 a$ \\
\hline & T4 (L+ 1/2 DV) & $73750 \pm 2956 a$ & $72579 \pm 3422 a$ & $1,67 \pm 0,97 a$ \\
\hline & T5 (L+ DV) & $77812 \pm 3109 a$ & $75156 \pm 3541 a$ & $3,48 \pm 1,49 a$ \\
\hline & Moyenne & $72141 \pm 5326$ & $69391 \pm 5715$ & $4,07 \pm 2,05$ \\
\hline & Probabilité (P) & 0,077 & 0,100 & 0,192 \\
\hline & Significativité & NS & NS & NS \\
\hline
\end{tabular}

Les moyennes dans la même colonne suivies de la même lettre ne sont pas significativement différentes au seuil de $5 \%$ (Test de Newman-Keuls). NS : non significative.

\section{Hauteur des plants de cotonnier}

Le tableau III présente la hauteur des plants de cotonnier à 30, 60, 90 et 120 jours après levée (JAL) pendant les deux années de culture. L'analyse de ce tableau a montré que 30 jours après levée (JAL), la hauteur des plants a varié de 24,93 à 29,48 cm, pour la $1^{\text {ère }}$ année et de 21,85 à $24,90 \mathrm{~cm}$, pour la $2^{\mathrm{e}}$ année. A ce stade de développement, l'analyse statistique n’a pas révélé de différence significative entre les différents traitements. Au $60^{\mathrm{e}}$ jour, la taille des plants, dans les deux campagnes (2018 et 2019), a fluctué respectivement entre 55,10 et $82,88 \mathrm{~cm}$, et entre 35,67 et $81,38 \mathrm{~cm}$. Au $90^{\mathrm{e}}$ jour, les hauteurs des plants ont varié de 65,50 à $109,85 \mathrm{~cm}$, pour la $1^{\text {ère }}$ année et de 50,38 et $97,25 \mathrm{~cm}$, pour la $2^{\mathrm{e}}$ année. Au $120^{\mathrm{e}}$ jour, la taille des plants a été comprise entre 74,05 et 118,23 cm la première année, et de 55,75 à 102,88 cm la deuxième année. Pour ces trois périodes, l'analyse statistique a révélé une différence très hautement significative entre les traitements. Pour les deux années de culture, c'est le traitement T5 (Légumineuse + Dose vulgarisée d'engrais) qui a eu les plus grands plants, avec des hauteurs supérieures à 100 $\mathrm{cm}$ à la récolte, tandis que le témoin absolu T1 a présenté les plus petits plants.

Tableau III. Hauteur des plants de cotonnier pendant les deux années de culture

\begin{tabular}{|c|c|c|c|c|c|}
\hline \multirow{2}{*}{$\begin{array}{l}\text { Anné } \\
\text { es }\end{array}$} & \multirow[b]{2}{*}{ Traitements } & \multicolumn{4}{|c|}{ Hauteur des plants } \\
\hline & & 30 JAL & $60 \mathrm{JAL}$ & $90 \mathrm{JAL}$ & $120 \mathrm{JAL}$ \\
\hline \multirow{6}{*}{$\begin{array}{l}2018- \\
2019\end{array}$} & $\mathrm{~T} 1(\mathrm{JN})$ & $25,18 \pm 2,88 a$ & $55,10 \pm 3,46 b$ & $65,50 \pm 5,49 c$ & $74,05 \pm 5,58 \mathrm{c}$ \\
\hline & $\mathrm{T} 2(\mathrm{JN}+\mathrm{DV})$ & $27,35 \pm 3,06 a$ & $76,08 \pm 12,36 a$ & $92,98 \pm 16,84 b$ & $96,23 \pm 15,75 b$ \\
\hline & T3 (L+ 0 DV) & $29,48 \pm 3,01 a$ & $69,78 \pm 10,07 \mathrm{ab}$ & $78,73 \pm 9,88 \mathrm{bc}$ & $85,38 \pm 7,97 \mathrm{bc}$ \\
\hline & $\mathrm{T} 4(\mathrm{~L}+1 / 2 \mathrm{DV})$ & $24,93 \pm 1,66 a$ & $68,50 \pm 7,40 \mathrm{ab}$ & $85,90 \pm 9,34 b$ & $91,78 \pm 12,29 \mathrm{bc}$ \\
\hline & T5 (L+ DV) & $29,18 \pm 6,32 a$ & $82,88 \pm 11,80 a$ & $109,85 \pm 8,83 a$ & $118,23 \pm 8,84 a$ \\
\hline & Moyenne & $27,22 \pm 3,85$ & $70,47 \pm 12,74$ & $86,59 \pm 17,90$ & $93,13 \pm 17,74$ \\
\hline
\end{tabular}




\begin{tabular}{|l|l|l|l|l|l|}
\hline & $\begin{array}{l}\text { Probabilité } \\
\text { (P) }\end{array}$ & $\mathbf{0 , 3 0 7}$ & $\mathbf{0 , 0 1 2}$ & $\mathbf{0 , 0 0 0}$ & $\mathbf{0 , 0 0 0}$ \\
\cline { 2 - 6 } & Significativité & NS & S & THS & THS \\
\hline \multirow{4}{*}{$\begin{array}{l}2019- \\
2020\end{array}$} & T1 (JN) & $21,98 \pm 1,96 \mathrm{a}$ & $35,67 \pm 3,61 \mathrm{c}$ & $50,38 \pm 3,29 \mathrm{c}$ & $55,75 \pm 4,65 \mathrm{c}$ \\
\cline { 2 - 6 } & T2 (JN + DV) & $24,73 \pm 0,63 \mathrm{a}$ & $57,20 \pm 2,85 \mathrm{~b}$ & $71,75 \pm 4,07 \mathrm{~b}$ & $75,25 \pm 3,54 \mathrm{bc}$ \\
\cline { 2 - 6 } & $\mathrm{T} 3(\mathrm{~L}+0 \mathrm{DV})$ & $21,85 \pm 1,49 \mathrm{a}$ & $61,30 \pm 5,24 \mathrm{~b}$ & $70,25 \pm 6,64 \mathrm{~b}$ & $79,25 \pm 6,11 \mathrm{abc}$ \\
\cline { 2 - 6 } & $\mathrm{T} 4(\mathrm{~L}+1 / 2 \mathrm{DV})$ & $24,90 \pm 1,63 \mathrm{a}$ & $81,38 \pm 8,50 \mathrm{a}$ & $94,50 \pm 8,99 \mathrm{a}$ & $98,75 \pm 9,40 \mathrm{ab}$ \\
\cline { 2 - 6 } & T5 (L+ DV) & $23,53 \pm 2,52 \mathrm{a}$ & $80,23 \pm 4,90 \mathrm{a}$ & $97,25 \pm 5,11 \mathrm{a}$ & $102,88 \pm 7,34 \mathrm{a}$ \\
\cline { 2 - 6 } & Moyenne & $23,40 \pm 0,760$ & $63,16 \pm 4,42$ & $76,83 \pm 4,63$ & $82,38 \pm 4,70$ \\
\cline { 2 - 6 } & $\begin{array}{l}\text { Probabilité } \\
\text { (P) }\end{array}$ & $\mathbf{0 , 6 1 4}$ & $\mathbf{0 , 0 0 0}$ & $\mathbf{0 , 0 0 0}$ & $\mathbf{0 , 0 0 0}$ \\
\cline { 2 - 6 } & Significativité & NS & THS & THS & THS \\
\hline
\end{tabular}

Les moyennes dans la même colonne suivies de la même lettre ne sont pas significativement différentes au seuil de 5\%. NS : non significative et THS : très hautement significative.

$>$ Effet du précédent arachide et de la fumure minérale sur la production du coton

\section{Nombre de capsules vertes par plant de cotonnier}

L'analyse des résultats consignés dans le tableau IV n'a révélé aucune différence significative entre les traitements, sur les deux ans. Pour la $1^{\text {ère }}$ campagne, le nombre de capsules à varié de 2,13 à 2,63 70 jours après levée (JAL) et de 0,70 à 2,12, pour le même stade phénologique. A $90 \mathrm{JAL}$, le nombre de capsules a été source de variabilité entre les traitements au cours des deux campagnes agricoles. Ce nombre a fluctué entre 4,95 et 10,23 pour la $1^{\text {ère }}$ campagne et entre 4,35 et 8,25 pour la $2^{\mathrm{e}}$. Les deux traitements fertilisés à dose complète d'engrais (T2 et T5) ont fourni plus de capsules la $1^{\text {ère }}$ année, tandis qu'en $2^{\mathrm{e}}$ année, c'est le traitement T4 (précédents arachides+ $1 / 2$ dose d'engrais) qui a produit plus de capsules. A 110JAL, le nombre moyen de capsules a varié de 3,45 à 7,03 et de 4,73 à 8,45 respectivement la $1^{\text {ère }}$ et la $2^{\mathrm{e}}$ année. Au plan statistique, le nombre de capsules a évolué selon les traitements sur les deux années de culture. Ce sont les précédents arachides fertilisés à différente dose d'engrais ( $\mathrm{T} 5$ pour la $1^{\text {ère }}$ année et $\mathrm{T} 4$ pour la $2^{\mathrm{e}}$ année) qui ont généré les nombres de capsules les plus importants.

Tableau IV. Nombre de capsules vertes par plant de cotonnier

\begin{tabular}{|c|c|c|c|c|}
\hline \multirow{3}{*}{ Années } & \multirow{3}{*}{ Traitements } & \multicolumn{3}{|c|}{ Capsules vertes / plant de cotonnier } \\
\cline { 3 - 5 } & T1 JAL & $\mathbf{9 0}$ JAL & $\mathbf{1 1 0}$ JAL \\
\hline \multirow{4}{*}{$2018-2019$} & $2,13 \pm 0,81 \mathrm{a}$ & $4,95 \pm 1,01 \mathrm{~b}$ & $3,45 \pm 0,63 \mathrm{~b}$ \\
\cline { 2 - 5 } & $\mathrm{T} 2(\mathrm{JN}+\mathrm{DV})$ & $2,63 \pm 1,3 \mathrm{a}$ & $9,6 \pm 2,64 \mathrm{a}$ & $6,1 \pm 1,54 \mathrm{a}$ \\
\cline { 2 - 5 } & $\mathrm{T} 3(\mathrm{~L}+0 \mathrm{DV})$ & $2,15 \pm 0,86 \mathrm{a}$ & $7,93 \pm 1,69 \mathrm{ab}$ & $5,75 \pm 1,14 \mathrm{a}$ \\
\cline { 2 - 5 } & $\mathrm{T} 4(\mathrm{~L}+1 / 2 \mathrm{DV})$ & $2,13 \pm 0,67 \mathrm{a}$ & $7,53 \pm 2,09 \mathrm{ab}$ & $5,73 \pm 0,64 \mathrm{a}$ \\
\cline { 2 - 5 } & $\mathrm{T} 5(\mathrm{~L}+\mathrm{DV})$ & $2,48 \pm 1,13 \mathrm{a}$ & $10,23 \pm 1,49 \mathrm{a}$ & $7,03 \pm 0,45 \mathrm{a}$ \\
\cline { 2 - 5 } & Moyenne & $\mathbf{2 , 3 0} \pm \mathbf{0 , 9 0}$ & $\mathbf{8 , 0 5} \pm \mathbf{2 , 5 2}$ & $\mathbf{5 , 6 1} \pm \mathbf{1 , 4 8}$ \\
\hline
\end{tabular}




\begin{tabular}{|c|c|c|c|c|}
\hline \multirow{4}{*}{$2019-2020$} & Probabilité (P) & $\mathbf{0 , 9 1 8}$ & $\mathbf{0 , 0 1 0}$ & $\mathbf{0 , 0 0 2}$ \\
\cline { 2 - 5 } & Significativité & NS & S & HS \\
\hline & T1 (JN) & $0,70 \pm 0,32 \mathrm{a}$ & $4,35 \pm 0,53 \mathrm{~b}$ & $4,73 \pm 0,55 \mathrm{~b}$ \\
\cline { 2 - 5 } & $\mathrm{T} 2(\mathrm{JN}+\mathrm{DV})$ & $1,58 \pm 0,19 \mathrm{a}$ & $7,35 \pm 0,858 \mathrm{a}$ & $7,38 \pm 0,63 \mathrm{ab}$ \\
\cline { 2 - 5 } & $\mathrm{T} 3(\mathrm{~L}+0 \mathrm{DV})$ & $1,40 \pm 0,33 \mathrm{a}$ & $6,73 \pm 0,17 \mathrm{a}$ & $6,38 \pm 0,456 \mathrm{ab}$ \\
\cline { 2 - 5 } & $\mathrm{T} 4(\mathrm{~L}+1 / 2 \mathrm{DV})$ & $1,05 \pm 0,22 \mathrm{a}$ & $8,25 \pm 1,1 \mathrm{a}$ & $8,45 \pm 1,11 \mathrm{a}$ \\
\cline { 2 - 5 } & $\mathrm{T} 5(\mathrm{~L}+\mathrm{DV})$ & $2,12 \pm 0,44 \mathrm{a}$ & $7,48 \pm 0,33 \mathrm{a}$ & $7,20 \pm 0,25 \mathrm{ab}$ \\
\cline { 2 - 5 } & Moyenne & $\mathbf{1 , 3 7} \pm \mathbf{0 , 1 7}$ & $\mathbf{6 , 8 3} \pm \mathbf{0 , 4 1}$ & $\mathbf{6 , 8 3} \pm \mathbf{0 , 3 9}$ \\
\cline { 2 - 5 } & Probabilité (P) & $\mathbf{0 , 0 5 7}$ & $\mathbf{0 , 0 1 1}$ & $\mathbf{0 , 0 1 0}$ \\
\cline { 2 - 5 } & Significativité & $\mathbf{N S}$ & $\mathbf{S}$ & $\mathbf{S}$ \\
\hline
\end{tabular}

Les moyennes dans la même colonne suivies de la même lettre ne sont pas significativement différentes au seuil de $5 \%$ (Test de Newman-Keuls). $N S$ : non significative, $S$ : significative.

\section{Rendement du coton-graine}

Dans le tableau $\mathbf{V}$ sont consignés les rendements en coton graine dans les essais arachide sur deux années de culture. Les résultats obtenus ont indiqué que les rendements ont varié en fonction des traitements. La $1^{\text {ère }}$ année, les rendements ont varié de 702,42 à 1423,15 kg/ha, avec une moyenne de 1146,09 kg/ha. Le précédent arachide fertilisé à dose complète d'engrais (T5) a été plus productif (1423 kg/ha). Le témoin (T1) a eu le rendement le plus faible. En comparaison au témoin T2 (pratique conventionnelle en milieu paysan), les arrières effets de l'arachide T5 (14,39\%) et T4 (3,78\%.) ont donné des rendements du coton-graine plus élevés. Avec le précèdent arachide sans engrais (T3), il y’a eu une diminution de rendement de 13,99 \% (Figure 3).

A la $2^{\mathrm{e}}$ année, les rendements ont varié de 578,13 à 1482,42 kg/ha, avec une moyenne de $1088 \mathrm{~kg} / \mathrm{ha}$. L'analyse statistique a révélé une différence très hautement significative entre les traitements. Le précédent arachide fertilisé à demi dose d'engrais (T4) a été plus productive $(1482,42 \mathrm{~kg} / \mathrm{ha})$ (Tableau V). L'effet de l'arachide a permis un gain de 42,05 \% en T5 et de $58,79 \%$ en T4 sur rendement du coton-graine, par rapport à la pratique conventionnelle en milieu paysan. Le précédent arachide sans engrais (T3) a connu une hausse de 19,67 \% (Figure 3).

Tableau V. Rendements du coton graine dans les essais arachide sur deux années de culture

\begin{tabular}{|l|l|l|}
\hline Traitements & $\begin{array}{l}\text { Rendement du coton-graine } \\
\text { (kg /ha) } \mathbf{1}^{\text {ere }} \text { année }\end{array}$ & $\begin{array}{l}\text { Rendement du coton-graine } \\
\text { (kg /ha) } \mathbf{2}^{\mathbf{e}} \text { année }\end{array}$ \\
\hline T1 (JN) & $702,42 \pm 81,42 \mathrm{~b}$ & $578,13 \pm 106,07 \mathrm{~d}$ \\
\hline T2 (JN + DV) & $1244,27 \pm 188,18 \mathrm{ab}$ & $933,59 \pm 34,57 \mathrm{c}$ \\
\hline T3 (L+ 0 DV) & $1070,50 \pm 179,19 \mathrm{ab}$ & $1117,19 \pm 104,67 \mathrm{bc}$ \\
\hline T4 (L+1/2 DV) & $1291,10 \pm 158,63 \mathrm{ab}$ & $1482,42 \pm 93,12 \mathrm{a}$ \\
\hline T5 (L+ DV) & $1423,15 \pm 162,47 \mathrm{a}$ & $1326,17 \pm 102,68 \mathrm{ab}$ \\
\hline
\end{tabular}




\begin{tabular}{|l|l|l|}
\hline Moyenne & $1146,09 \pm 85,06$ & $1088,50 \pm 81,10$ \\
\hline Probabilité (P) & $\mathbf{0 , 0 4 9}$ & $\mathbf{0 , 0 0 0}$ \\
\hline CV \% & 7,42 & 7,45 \\
\hline Significativité & S & THS \\
\hline
\end{tabular}

Les moyennes dans la même colonne suivies de la même lettre ne sont pas significativement différentes au seuil de $5 \%$ (Test de Newman-Keuls). S : significative, THS : très hautement significative.

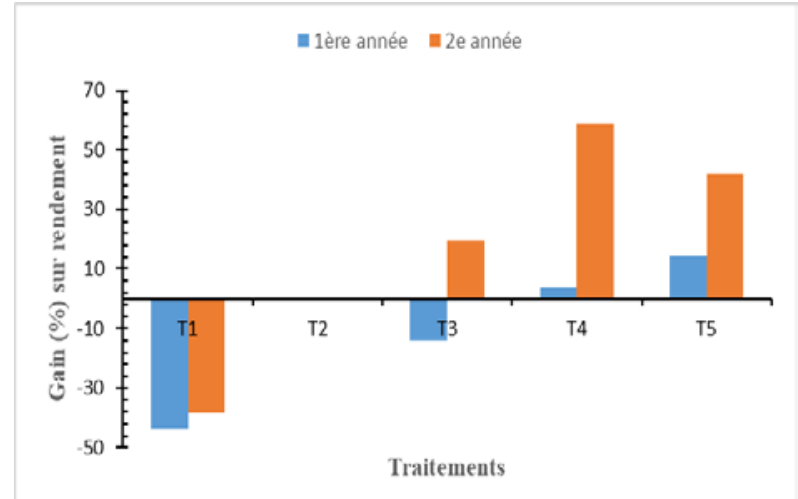

Figure 3. Gain du coton graine sur les deux années de culture

\section{Efficience agronomique des fertilisants}

La figure 4 indique l'efficience agronomique des fertilisants appliqués sur les plants de cotonnier pendant deux années de culture. D’après cette figure, l'efficience agronomique des fertilisant a varié de 0,939 à 4,796 en $1^{\text {ère }}$ année et de 1,287 à 3,15 la $2^{\mathrm{e}}$ année. Il a été noté que, le traitement T2 (pratique conventionnelle) a occasionné une forte efficience contrairement aux précédents arachides (T3, T4 et T5) qui en ont obtenu les plus faibles indépendamment des années de culture.

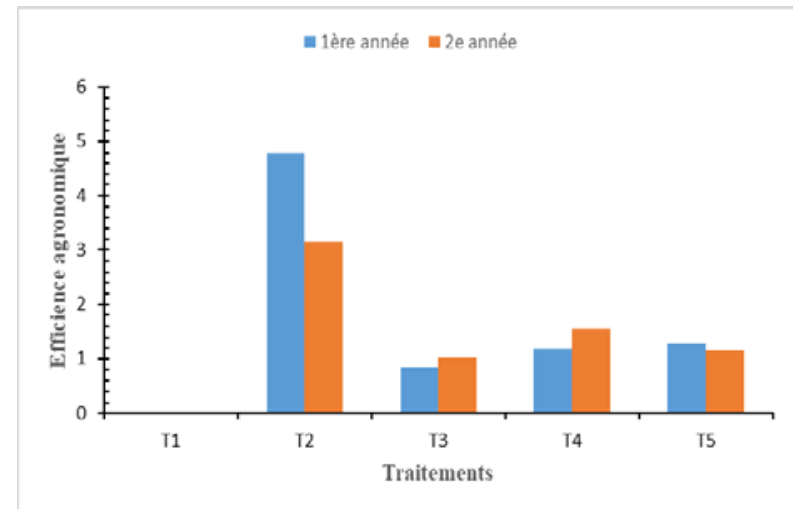

Figure 4. Efficience agronomique du coton graine sur les deux années de culture 


\section{Discussion}

\section{Effet du précédent arachide et de la fumure minérale sur les paramètres agro-morphologiques du cotonnier}

En ce qui concerne le taux de mortalité, les précédent arachide (T3, T4 et T5) ont obtenu les faibles taux. Ces traitements ont probablement fourni les éléments minéraux nécessaire et en quantité suffisante pour minimiser la compétition entre les plants. De plus, il est possible que la décomposition de la matière organique ait amélioré la structure du sol. Koné et al., (2009) puis Badou et al., (2013), ont fait le même constat en révélant que la structure du sol s'améliore par la décomposition de la biomasse dans le sol. Kouelo et al., (2012), affirme que les techniques culturales les plus recommandées pour une productivité soutenue, sont le travail du sol et la fertilisation organique.

De façon générale, l'analyse statistique a révélé une différence significative entre les traitements, concernant la croissance en hauteur des plants. Les précédents arachides fertilisés à différentes doses d'engrais (T4 et T5) ont eu de grands plants. Le développement végétatif des cotonniers pourrait être dû aux éléments minéraux, que sont le phosphore et l'azote qui seraient en grande quantité dans le sol. Ces résultats confirment celui de Yoboue, (2015), qui a noté que le précédent arachide associé à l'engrais NPK et l'Urée assure une bonne croissance végétative du cotonnier.

\section{Effet du précédent arachide et de la fumure minérale sur le rendement}

Les précédents arachides fertilisés à différentes doses d'engrais (T4 et T5) ont eu plus de capsules. Cela est dû à la forte teneur de minéraux que disposerait les sols de ces traitements. Ces résultats rejoignent ceux de Ilboudo (1997) dans son étude sur le cotonnier, a montré que le gain sur capsules peut aller jusqu'à 19 p.c. quand on associe à l'engrais coton du fumier.

Les rendements du coton-graine obtenus ont montré l'effet bénéfique du précèdent arachide. En effet, les traitements (T4) et (T5) ont été significativement plus productifs avec plus de $1400 \mathrm{~kg} / \mathrm{ha}$ de coton-graine. Par rapport à la pratique conventionnelle, sur les deux campagnes, la culture d'arachide a permis d'améliorer le rendement du coton-graine de 14,39 \% et de 42,05 \% dans le traitement T5. Elle a permis également d'obtenir un gain de 58,79 \% avec T4 dans la seconde campagne. Les résidus de l'arachide laissés sur place ont restitué au sol des minéraux, surtout l'azote, qui joue, en effet, un rôle déterminant sur le rendement (Souley et al, 2020). En plus de la matière organique issue de la décomposition de la biomasse de l'arachide, il y a eu aussi l'azote résiduel laissé dans le sol en fin de cycle de l'arachide. Après la récolte de l'arachide, il est resté encore de l'azote dans le sol exploitable par le cotonnier (Coulibaly et al, 2021). C'est donc dire que le cotonnier auquel les traitements T4 et T5 ont été appliqués, ont bénéficié de trois sources de minéraux; en l'occurrence ceux issus du sol, des rhizobiums et de la 
décomposition de la matière organique. Les résultats concordent avec les études faites par Bado (2002) et Yoboué (2015), qui ont montré que les rotations comportant les précédents arachide associés à la fumure minérale augmentaient significativement les rendements du sorgho et du coton. Par ailleurs Bado (2002) soutient que les rendements en grains et en paille du sorgho ont augmenté respectivement de 70 et 100 \%, et même ont été doublés lorsqu'il est précédé par les légumineuses alimentaires, en comparaison à la monoculture.

Les rendements obtenus avec le traitement $\mathrm{T} 4$ révèlent que l'utilisation de la fumure minérale peut être réduite par le précédent arachide, car il garantirait deux sources de minéraux à savoir l'action des bactéries et la biomasse de l'arachide. Ces résultats rejoignent ceux de Konate et al, (2012), qui attestent que l'utilisation de légumineuses alimentaires tels que le soja et le niébé comme précédents culturaux, permet de réduire du quart pour le niébé ou de la moitié pour le soja la dose d'engrais vulgarisée. Voisin et al, (2015) affirment également que les effets d'une culture de légumineuse sur les flux azotés à court et moyen termes permettent une réduction de la fertilisation azotée sur la culture suivante.

Les forts gains obtenus dans les précédents arachides la $2^{\mathrm{e}}$ année contrairement à la $1^{\mathrm{e}}$ année, pourraient s'expliquer par le fait que la quantité de biomasse sèche produite par l'arachide à la $2^{\mathrm{e}}$ année $(10083 \mathrm{~kg} / \mathrm{ha})$ a été plus que celle produite la $1^{\text {ère }}$ année $(8469 \mathrm{~kg} / \mathrm{ha})$. De ce fait, la teneur en éléments minéraux, dont l'azote, mobilisé dans le sol la deuxième année a été plus élevée. La quantité de la biomasse produite par l'arachide a assuré une couverture optimale, qui a dû favoriser l'amélioration de l'humidité du sol. Ces conditions favorables ont entrainé un bon développement végétatif et un gain sur rendement de 19,67 \% en utilisant le traitement T3. Voisin et al., (2015) ont montré que par leur capacité à s'associer avec des bactéries du sol, les légumineuses ont la particularité de pouvoir être cultivées sans utiliser d'engrais azotés. Elles sont une source d'azote pour les cultures qui leur sont associées ou pour celles qui sont cultivées à leur suite. Nos résultats corroborent ceux de Konate et al., (2013) qui, dans leur étude sur la culture du riz pluviale, ont montré qu'une bonne couverture du sol pourrait influencés les paramètres de croissance des plantes. Ces auteurs poursuivent en justifiant que la biomasse aérienne sèche produite par le soja s'est distingué, avec $410 \mathrm{~g} / \mathrm{m}^{2}$ contre 209,38 g/m2 pour le niébé. Ce qui a permis à l'antécédent soja, d'avoir un rendement moyen $(2445,82 \mathrm{~kg} / \mathrm{ha}$ de riz paddy) plus élevé que celui de l'antécédent niébé (2421,04 kg/ha de riz paddy). Carsky et al., (2003) ; Affokpon et al.,(2013) et Badou et al., (2013) ont aussi montré au Burkina Faso et Benin que, par la quantité de biomasse produite, les légumineuses à graines (arachide, niébé ou soja) ont eu un effet positif sur le rendement de céréale (maïs et mil). 
Les résultats obtenus avec l'efficience agronomique ont montré une baisse dans les traitements (T3), (T4) et (T5). Cela est dû d'une part à la forte utilisation de minéraux NPK (organique) mobilisé par la biomasse d'arachide dans ces traitements. Et d'autre part aux faibles rendements obtenu (traitement T3). Ces résultats concordent avec ceux de Akassimadou et al., (2017) qui ont montré que l'efficience agronomique suit une tendance à la baisse au fur et à mesure que la dose de fertilisants augmente.

\section{Conclusion}

Les traitements T4 et T5 qui sont les précédents arachides associés à différentes doses d'engrais minéraux influencent positivement sur les paramètres agro-morphologique du cotonnier et le rendement (gain de 58,79\% et 42,05 \%) en comparaison à T2 (précédent jachère + dose complète engrais). Et même pour les traitements sans fumure minéral, T3 (précédent arachides) a eu des résultats satisfaisants. Cette étude a révélé que la légumineuse arachide, utilisée comme précédent cultural dans la culture du coton a été une source de matières organiques susceptibles de restaurer les sols sous cotonnier et en les enrichissant en éléments minéraux. La biomasse sèche produite améliore le rendement en coton-graine et permet de réduire l'utilisation de fertilisant minéral. Cette pratique culturale s'avère d'autant plus intéressante, qu'elle aiderait les paysans à réduire les charges en engrais chimique, tout en préservant l'environnement.

\section{Remerciements}

Nos remerciements au Centre National de Recherche Agronomique (CNRA) pour avoir financé cette étude à travers le programme coton.

\section{References:}

1. Adingra Y. K., 2011. Coton Africain : Défis, Enjeux et Perspectives. Document de travail Université Paris1 Panthéon -Sorbonne Rencontre panafricaine sur le coton, 21p.

2. Affokpon, J. A. P. Djènontin, A. G. Zoffoun, M. C. Allagbé, T. P. Akondé, K. Aïhou, G. Kpagbin, H. Gotoechan-Hodonou, J. Détongnon et G. A. Mensah, 2013. Effets des variétés de niébé à buts multiples comme précédent cultural sur le rendement du maïs cultivé sur terres de barre dégradées au Sud-Bénin. Bulletin de la Recherche Agronomique du Bénin (BRAB) Numéro spécial fertilité du maïs p5868.

3. Akassimadou F. E., Hien M. P., Bouadou Oi F. B., Bolou Bi E. B., Bongoua J. A., Ettien J.-B. D., Yao-kouame A., 2017. Efficiences des nutriments $\mathrm{P}$ Et $\mathrm{K}$ en riziculture irriguée dans un bas-fond secondaire 
en zone de savane Guinéenne de la Côte d'Ivoire. European Scientific Journal edition Vol.13, No.36 : 432-453.

Doi : http://dx.doi.org/10.19044/esj.2017.v13n36p432.

4. Akédrin T. N., N’Guessan K., Aké A. E. et Aké S., 2010. Effet de Légumineuses herbacées ou subligneuses sur la productivité du maïs. (JAPS) Journal of Animal \& Plant Sciences. Vol. 8: 953- 963. Université de Cocody-Abidjan (Côte-d'Ivoire).

5. Anonyme, 2013. Système de semis direct sous couverture végétale. Manuel de bonnes pratiques agricoles sur le coton, COTON-4. 85 p.

6. Bado B.V., 2002. Rôle des légumineuses sur la fertilité du sols ferralitiques tropicaux des zones guinéennes et soudanniennes du Bourkina Fasso. Thèse de doctorat à l'université de Laval, Québec ; 166p.

7. Badou A., Akondé P. T., Adjanohoun A., Adjé I. T., Aïhou K. et Igué A. M., 2013. Effets de différents modes de gestion des résidus de soja sur le rendement du maïs dans deux zones agroécologiques du CentreBénin. Bulletin de la Recherche Agronomique du Bénin (BRAB) Numéro spécial Fertilité du maïs; 5p.

8. Berti F., Hofs J. L., Zagbaï H. S. et Lebailly P. (2006). Le coton dans le monde, place du coton africain et principaux enjeux. Biotechnologie, Agronomie, Société et Environnement, 10 (4) : 271280.

9. Carsky R.J., Douthwaite B., Mariyong V.M., Sanginga N., Schulz S., VanlauweB., Diels J. et Keatinge J.D.H., 2003. Amélioration de la gestion des sols par l'introduction de légumineuses dans les systèmes céréaliers des savanes africaines. Cah. Agri. 12 : 227-233.

10. Coulibaly K., 2012. Analyse des facteurs de variabilité des performances agronomiques et économiques des cultures et de l'évolution de la fertilité des sols dans les systèmes agropastoraux en milieu soudanien du Burkina Faso : approche expérimentale chez et par les paysans. Doctorat en Développement rural de l'Université Polytechnique de Bobo Dioulasso. Spécialité : sciences des sols. 149 p.

11. Coulibaly K., Vall E., Autfray P. et Sedogo P. M., 2012. Performance technico-économique des associations maïs/niébé et maïs/mucuna en situation réelle de culture au Burkina Faso: potentiels et contraintes. Tropicultura, , 30 (3) : 147-154.

12. Coulibaly N.D., Ossey C.L., Gadji A.G., N’Gbesso M.F.D.P., Fondio L. \& Soro O.T. (2021). Etude De L’arrière Effet Des Légumineuses Alimentaires Sur La Productivité Des Légumes : Cas De La Tomate (Solanum Lycopersicum), Cultivée Dans La Localité De Bouaké Au 
Centre De La Côte d’Ivoire. European Scientific Journal, ESJ, 17(21), 125. https://doi.org/10.19044/esj.2021.v17n21p125

13. FAO, 2015. De la Déclaration d'Abuja sur les engrais au cadre de gestion durable des sols pour la sécurité alimentaire et nutritionnelle en Afrique à l'horizon 2030. Stimuler les sols africains ; 16p.

14. Gbakatchetche H., Sanogo S.; Camara M., Bouet A., Keli J.Z., 2010. Effet du paillage par des résidus de pois d'angole (cajanus cajan l.) sur le rendement du riz (oryza sativa) pluvial en zone forestière de Côte d'Ivoire. Agronomie Africaine 22 (2) : 131 - 137.

15. Hinsinger P., 2012. Les cultures associées. in :Les Cultures Associées Céréale / Légumineuse En agriculture «bas intrants » dans le Sud de la France ; 27p.

16. Ilboudo O., 1997. Effet des fumures de fond sur l'acidité du sol et la croissance du cotonnier. Mémoire pour obtention du diplôme d'ingénieur à l'université polytechnique de Bobo, Burkina ; 79p.

17. Konate Z., Gala Bi T. J., Messoum F. G., Sekou A., Yao KA., Camara M., Keli Z. J., 2012. Alternatives à la fertilisation minérale des sols en riziculture pluviale de plateau : apports des cultures du soja et du niébé dans la fertilité d'un ferralsol hyperdystrique au Centre-ouest de la Côte d'Ivoire. Journal of Applied Biosciences 54: 3859 - 3869.

18. Konate Z, Messoum FG, Sekou A, Yao KA, Camara M, Keli ZJ. 2013. Effets des cultures de soja (Glycine max) et de niébé (Vigna unguiculata) sur la densité apparente et la teneur en eau des sols et sur la productivité du riz pluvial de plateau sur ferralsol hyperdystrique: cas de Gagnoa, au Centre Ouest de la Côte d’Ivoire. Int. J. Biol. Chem. Sci. 7(1) : 47-59. DOI : http://dx.doi.org/10.4314/ijbcs.v7i1.5

19. Koné B., Diatta S., Saïdou A., Akintayo I., Cissé B., 2009. Réponses des variétés interspécifiques du riz de plateau aux applications de phosphate en zone de forêt au Nigeria. Canadian Journal of Soil Science: 555-565.

20. Kouelo A. F., Badou A., Houngnandan P., Francisco M. M. F., Gnimassoun C. J.-B., Sochime D. J., 2012. Impact du travail du sol et de la fertilisation minérale sur la productivité de Macrotyloma geocarpum (Harms) Maréchal \& Baudet au centre du Bénin. Journal of Applied Biosciences 51: 3625- 3632

21. N'Goran K. E., Kassin K. E., Zohouri G. P., N’Gbesso M. F. D. P., Yoro G. R., 2011. Performances agronomiques des associations culturales igname-légumineuses alimentaires dans le Centre-ouest de la Côte d'Ivoire. Journal of Applied Biosciences 43: 2915 - 2923.

22. N’Goran K. E., Kassin K. E., Brou K. J., Gustave F. M., Kouamé B., N'guessan D. B., 2018. Diagnostic de l'Etat de Fertilité des Sols Sous 
Culture Cotonnière Dans les Principaux Bassins de Production de Côte d'Ivoire. European Scientific Journal, 14 (33) : 221-238.

Doi: http://dx.doi.org/10.19044/esj.2018.v14n33p221.

23. N'Goran K. E., 2008. Effet des légumineuses à grains et des plantes de couverture sur la fertilité des sols et la production de l'igname en zone soudano-guinéenne de Cote d'Ivoire. Mémoire de thèse à l'Université de Cocody ; 183p.

24. Ochou O. G., Koto E., N’Guessan E., Ouraga Y., Tehia K. E., Kouadio N. N. et Angbo S., 2003. Analyse et Propositions pour le Programme Coton de 2ème Génération., Période 2004-2007, Sous-commission Coton. Document CNRA ; 14 p.

25. Ouattara N., 2001. Note thématique sur les ressources génétiques forestières. Situation des ressources génétiques forestières de la côte d'ivoire (zone de savanes) ; 43 p.

26. Samba T, Minamba B, Birama SC, Adama C. 2013. Amélioration de la gestion de la fertilité des sols et celle des cultures dans les zones sahéliennes de l'Afrique de l'Ouest : une condition sine qua none pour l'augmentation de la productivité et de la durabilité des systèmes de culture à base de mil. Research Gate; 26 p.

https://www.researchgate.net/publication /237827087.

27. Souley M. S., Addam K. S., Mourou B., Jens B. A., 2020. Effets de la Fertilisation à Base de la Biomasse du Sida cordifolia L. sur les Performances Agronomiques et la Rentabilité Économique de la Tomate (Lycopersicum esculentum Mill.) en Culture Irriguée. European Scientific Journal, 16 (3) : 127-150.

Doi:10.19044/esj.2020.v16n3p127.

28. Vertès F., Jeuffroy M.-H., Louarn G., Voisin A.-S., Justes E., 2015. Légumineuses et prairies temporaires : des fournitures d'azote pour les rotations. Fourrages (2015), 221-232.

29. Voisin A.-S., Cellier P., Jeuffoy M.-H., 2015. Fonctionnement de la symbiose fixatrice de $\mathrm{N}$ des légumineuses à graines : Impacts Agronomiques et Environnementaux. Innovations Agronomiques 43 : 139-160.

30. Yoboué A.N. Epse K. 2015. Effets des précédents arachides (Arachis hypogaea) et de la fumure minérale sur la culture du Coton (Gossypium hirsutum L.).memoire de master en Sciences et Gestion de l'Environnement à l' Université Nangui-Abrogoua ; 45p.

31. Yoro G. R., 2000. Les principaux sols de côte d'ivoire et leur corrélation avec les groupes de références de la Base Mondial des données de sols (WRB) ; 19p. 